\title{
Fathers' perception of child's weight and paternal feeding practices in relation to child's gender
}

\author{
Stephanie Rahill, Aileen Kennedy and John Kearney \\ Technological University of Dublin, Dublin, Ireland
}

\section{Abstract}

The role of fathers in child rearing has changed in recent years due to an increase in maternal employment, which has increased the prevalence of co-parenting. It is important therefore to establish the role fathers have in feeding their child and how their attitudes and perceptions may influence the strategies they employ during mealtimes. In addition, research suggests that maternal and paternal feeding practices are associated with child's gender and weight status. Therefore, the aim of this research was to investigate the association between fathers' perception of child's weight and paternal non-responsive and structure-related feeding practices, and the extent to which this is mediated by a child's gender.

Cross-sectional data from an Irish sample of fathers, who completed an online survey based on one of their children aged 5-12 years old $(\mathrm{n}=155)$. The Feeding Practices and Structure Questionnaire (FPSQ) assessed paternal feeding practices, and it was validated for use within this sample. A 5-point Likert scale response ranging from Very Underweight to Very Overweight to the question "Do you think your child is . . ?" assessed fathers' perception of child's weight. Associations were assessed using multiple linear regressions.

Fathers who perceived their sons as underweight reported higher levels of rewarding the child to eat $(\beta=.24 ; p=0.04)$ and overt restriction $(\beta=.27 ; p=0.03)$ than fathers who perceived their sons to be normal weight. Fathers who perceived their sons as overweight reported higher levels of overt restriction $(\beta=.26 ; p=0.04)$ than fathers who perceived their son to be normal weight. In relation to daughters, fathers who perceived them as underweight reported higher levels of both reward for behaviour $(\beta=.28 ; \mathrm{p}=0.006)$ and reward for eating $(\beta=.20 ; p=0.04)$, while fathers who perceived their daughters as overweight reported lower levels of structured mealtimes $(\beta=-.27 ; p=0.012)$ compared to fathers who perceived their daughter as normal weight.

Findings suggest that paternal perception of child's weight influences the type of feeding practices utilised by fathers, with the child's gender a mediating influence on reported specific feeding practices. Overall, fathers who perceive their son or daughter to be underweight or overweight appear to engage in more non-responsive and less structure-related feeding practices than fathers who perceive their child to be normal weight.

\section{Conflict of Interest}

"There is no conflict of interest". 